\title{
Ferromagnetic, Ferrimagnetic and Spin-wave Resonances in GaMnAs Layers
}

\author{
O. Fedorych ${ }^{a}$, M. BYszeWski ${ }^{a, b}$, Z. WILAMOWSKI ${ }^{a, b, *}$, \\ M. POTEMSKI ${ }^{b}$ AND J. SADOWSKI ${ }^{a, c, d}$ \\ ${ }^{a}$ Institute of Physics, Polish Academy of Sciences \\ al. Lotników 32/46, 02-668 Warsaw, Poland \\ ${ }^{b}$ Grenoble High Magnetic Field Laboratory \\ MPI/CNRS, BP 166, 38042 Grenoble, France \\ ${ }^{c}$ Oersted Laborartory, Copenhagen University, 2100 Copenhagen, Denmark \\ ${ }^{d}$ Department of Experimental Physics \\ Chalmers University of Technology and Göteborg University, Sweden

\begin{abstract}
Molecular beam epitaxy grown $\mathrm{Ga}_{1-x} \mathrm{Mn}_{x}$ As layers were investigated by means of magnetic resonances. With an increase in Mn concentration, $x$, the spectrum changes from the (i) paramagnetic one, with resolved fine and hyperfine structures, typical of $S=5 / 2$ spin of substitutional $\mathrm{Mn}^{2+}$ ions, for very diluted alloy, via (ii) paramagnetic spectrum, where the fine and hyperfine structures are averaged by a long range $\mathrm{Mn}^{2+}-\mathrm{Mn}^{2+}$ exchange coupling, (iii) single, isotropic line of ferromagnetic resonance. Insulator to metal transition is accompanied with occurrence of (iv) a very complex spectrum of the ferrimagnetic resonance, accompanied with the well-resolved spin wave resonance. Reentrance to insulator phase for the most condensed alloys is accompanied with the reentrance to (v) ferromagnetic phase. The data confirm that the effective mass holes transfer the exchange interaction between localized $\mathrm{Mn}^{2+}$ spins.
\end{abstract}

PACS numbers: 71.55.Eq, 76.30.Fc, 75.50.Pp, 75.30.Gw

\section{Introduction}

$\mathrm{Ga}_{1-x} \mathrm{Mn}_{x}$ As is a canonical magnetic semiconductor which shows ferromagnetic properties [1-4]. It is one of the most promising diluted ferromagnets which

*corresponding author; e-mail: wilamz@ifpan.edu.pl 
can be applied in magnetic semiconductor devices. The ferromagnetic phase occurs already for $x=0.015$, and for $x=0.04$, the Curie temperature reaches $110 \mathrm{~K}$. Some logical elements, where the magnetic phase is switched on by an electric gate, have already been built $[3,4]$.

The properties of $\mathrm{Ga}_{1-x} \mathrm{Mn}_{x}$ As are investigated in many laboratories. Several models for the explanation of the magnetic properties in very diluted alloys were elaborated [5-15]. Nevertheless, some basic questions still remain open.

First of all, there is an open controversy on the ground state of Mn acceptor. Some models assume that the ground state corresponds to the $\mathrm{Mn}^{2+}$ ion with the $3 d^{5}$ configuration, characterized by $S=5 / 2$, and an effective mass hole [5-14]. But another authors assume an occurrence of partially filled $\mathrm{Mn}^{2+/ 3+}$ impurity band [15]. In the former case the $\mathrm{Mn}-\mathrm{Mn}$ exchange interaction is consequently assumed to be mediated by effective mass electrons, i.e. by RKKY-like interaction while in the second case the double exchange is postulated as the dominant exchange mechanism.

The role of the chemical disorder caused by the random distribution of the Mn ions is another open question. Theoretical models predict considerable fluctuation of the local density of $\mathrm{Mn}$ ions and the related fluctuations of the hole density. A noncollinear structure of $\mathrm{Mn}$ ions is theoretically predicted [7, 13, 14]. From that point of view it is not obvious if the real long range ferromagnetic order can occur in these very diluted alloys.

Finally, the origin of the magnetic anisotropy remain unclear. There is a class of $\mathrm{Ga}_{1-x} \mathrm{Mn}_{x}$ As samples which shows almost isotropic properties, but another class exhibits a strong magnetic anisotropy [2, 11, 12, 16].

Our systematic investigations of $\mathrm{Ga}_{1-x} \mathrm{Mn}_{x}$ As layers by means of magnetic resonances bring some answers on the listed question. They prove occurrence of $\mathrm{Mn}^{2+}$ ions coupled by effective mass holes. Different magnetic phases were found in insulating and metallic phases. In the insulating phase the $S=5 / 2$ spins form an isotropic ferromagnetic order. The holes are localized and their spatial distribution, and generally the spatial distribution of the ferromagnetic phase, are not homogeneous. In contrast, in the metallic phase, the extended states of holes lead to magnetically homogeneous phase. Here, the spin waves can easily propagate, the holes form their own macroscopic magnetic moment leading to a ferrimagnetic order. The magnetic anisotropy of the conducting holes results in the strong magnetic anisotropy of the ferrimagnetic phase.

\section{Experimental details}

The samples were grown by low temperature molecular beam epitaxy (LT MBE) [17]. The calibration of $\mathrm{Ga}$ and Mn sources allows us to estimate the Mn content with an accuracy better than $0.1 \%$. The composition of samples with low Mn concentrations was verified by SIMS measurements and by the integrated 
amplitude of electron paramagnetic resonance (EPR) signal. The thickness of low concentration samples was about 2 micrometers and of high concentration ones was about 1 micrometer. A wide range of the Mn composition was examined. The lowest composition is $x=10^{-5}$, the highest is $x=0.08$. The $\mathrm{X}$-ray diffraction (XRD) measurements showed that all samples with Mn content from $10^{-5}$ to $10^{-3}$ are coherently strained by the GaAs (100) substrate.

The measurement was done in the commercial X-band (9.4 GHz) BRUKER EPR spectrometer in the Institute of Physics in Warsaw. Some samples were additionally measured in a high frequency EPR spectrometer in HMFL, Grenoble. The measurement frequency was tuned in the range of 40-60 GHz and magnetic field up to $18 \mathrm{~T}$. In Fig. 1 various types of magnetic resonance spectra, measured at a temperature of $T=2 \mathrm{~K}$, for five different $\mathrm{Mn}$ concentrations are presented.

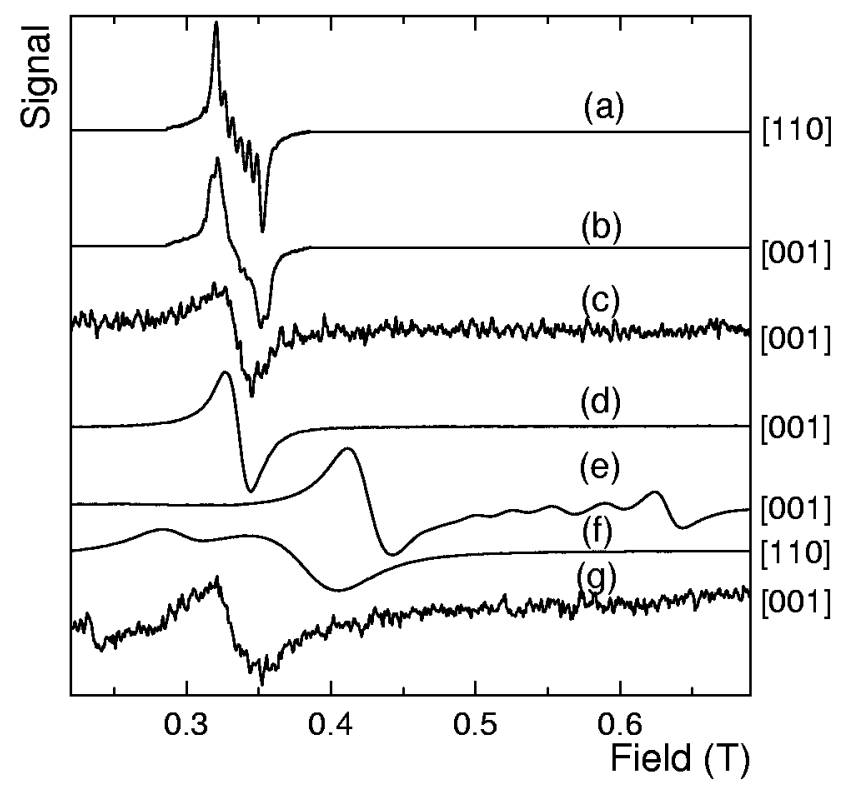

Fig. 1. The spectra of magnetic resonance of $\mathrm{Ga}_{1-x} \mathrm{Mn}_{x} \mathrm{As}$ measured at $T=2 \mathrm{~K}$, for various alloy compositions $x$. The resolved fine and hyperfine structure seen in the spectra (a), (b) proves that in a very diluted alloy the Mn impurity is not coupled by the exchange interaction. The spectrum (a) corresponds to in-plane and the spectrum (b) for perpendicular orientation of the applied field. The spectrum (c) shows the exchange narrowing of the fine and hyperfine structures caused by an occurrence of effective mass holes [16]. The spectra (d) and (g) are the ferromagnetic resonance which occurs in the insulating samples, while the spectra (e) and (f) stand for ferrimagnetic resonance occurring in metallic samples. The wavy structure at high magnetic field in the spectrum (e) is the spin waves resonance. The single line at $B=0.43 \mathrm{~T}$ is a new type of unrecognized excitation in a diluted ferrimagnetic semimetal. 
The spectra (a), (b), and (c) correspond to a paramagnetic phase. For very diluted samples well-resolved six lines of the hyperfine structure, caused by the coupling of $S=5 / 2$ electronic spin to $I=5 / 2$ nuclear spin, and weakly resolved five lines of a fine structure is observed. With an increase in Mn concentration, the exchange coupling via the effective mass holes bound on the acceptor states leads to the exchange narrowing of fine and hyperfine structure. The spectrum $(d)$ is an isotropic line of the ferromagnetic resonance, while the complex spectrum shown in Figs. 1e and $f$ stand for ferrimagnetic resonance observed in metallic samples, measured for two different directions of the applied magnetic field. Finally, the spectrum ( $\mathrm{g}$ ) is the ferromagnetic resonance which occurs in the insulating samples with a high Mn concentration.

\section{The paramagnetic resonance}

The paramagnetic resonance in $\mathrm{Ga}_{1-x} \mathrm{Mn}_{x} \mathrm{As}$ was investigated by several authors. Almeleh and Goldstein [18] observed the spectrum of $\mathrm{Mn}^{2+}$ ions in very diluted bulk samples. They evaluated hyperfine structure and estimated the upper limit of the cubic crystal field fine splitting. In addition to the spectrum typical of $3 d^{5}$ configuration of $\mathrm{Mn}^{2+}$ ion, characterized by $g$-factor $g=2$, Schneider et al. [19], found the paramagnetic resonance at $g=2.77$. They attributed this resonance to $S=1$ effective spin of the neutral acceptor originating from the coupling of the $S=5 / 2$ spin with $j=3 / 2$ magnetic moment of effective mass acceptor hole, bound on $\mathrm{Mn}^{2+}$ core. Szczytko et al. [20, 21] investigated MBE grown layers. They found the paramagnetic resonance similar to that measured by Almeleh and Goldstein [18], they observed the single line paramagnetic and ferromagnetic resonances (see Figs. $1 \mathrm{~b}$ and $\mathrm{c}$ ), but in the MBE layers they did not find any resonance which could be attributed to the neutral acceptor [19].

Our earlier systematic investigations of MBE grown $\mathrm{Ga}_{1-x} \mathrm{Mn}_{x}$ As layers [16] are in accordance with the results of Szczytko et al. [20, 21]. In spite of a very careful investigation of the variation of the EPR spectra with a change of $\mathrm{Mn}$ concentration we did not find any resonance of neutral acceptors. The high quality samples allow us to resolve the fine structure of the EPR spectrum. In consequence we were able to evaluate the single ion, crystal field anisotropy and to analyze the line broadening due to the local strain. We find that the cubic component of the crystal field is very small, the corresponding parameter of spin Hamiltonian $|a|=(14.1 \pm 0.3) \times 10^{-4} \mathrm{~cm}^{-1}$. The axial strain caused by a difference of the lattice constants between the GaAs substrate and $\mathrm{Ga}_{1-x} \mathrm{Mn}_{x}$ As layer effects the axial component of the crystal field seen by $\mathrm{Mn}^{2+}$ ion. But this origin of the magnetic anisotropy is definitely too small to be responsible for the magnetic anisotropy observed in ferrimagnetic $\mathrm{Ga}_{1-x} \mathrm{Mn}_{x}$ As. In the concentration range, where the fine structure is resolved, the axial component of the anisotropy field does not exceed $0.7 \mathrm{mT}$. 
The analysis of the variation EPR spectrum with the Mn concentration leads to the conclusions that the exchange narrowing of fine and hyperfine structures is not uniform for all $\mathrm{Mn}^{2+}$ spins. With the increase in doping, when $\mathrm{Mn}$ and holes concentrations increase, the fraction of $\mathrm{Mn}^{2+}$ spins which are coupled to each other increases, but the rest of $\mathrm{Mn}^{2+}$ spins remains unaffected by carries and by neighboring $\mathrm{Mn}^{2+}$ spins. This leads to the conclusion that the spatial distribution of carriers is not homogeneous. The simple modeling of the volume covered by carriers, transferring the exchange interaction, shows that the mean interacting radius coincides with the Bohr radius of acceptor states $[9,16]$.

The results of EPR proves that the magnetic moments observed in the paramagnetic phase originates from $\mathrm{Mn}^{2+}$. Neither resonance of carriers spin, nor of insulated neutral acceptor is observed. The difference between the MBE grown layer and the bulk crystal is probably caused by the presence of a high concentration of the donor-like native defects in the low temperature MBE samples. The presence of donors causes that the weakly doped samples, with Mn concentration $x<2 \times 10^{-4}$, are fully compensated. No effective mass hole occurs and all $\mathrm{Mn}$ dopants are in $\mathrm{Mn}^{2+}$ state. When, for the Mn concentration of about $x>2 \times 10^{-4}$ the native donors become overcompensated by $\mathrm{Mn}$ acceptors and the effective mass holes appear, the $\mathrm{Mn}-\mathrm{Mn}$ distance is too small to form the individual neutral acceptor states. The mean $\mathrm{Mn}-\mathrm{Mn}$ distance (about $6 \mathrm{~nm}$ ) is still considerably greater as compared to the Bohr radius, but the holes are preferably captured by the closely distributed $\mathrm{Mn}^{2+}$ clusters where the fluctuating Coulomb potential has its minima.

We carefully looked for the EPR signal which could be attributed to the spectrum of $\mathrm{Mn}^{3+}$, but up to frequency of $60 \mathrm{GHz}$ and magnetic field of $18 \mathrm{~T}$ no corresponding signal has been found.

\section{Ferromagnetic resonance}

The transition from paramagnetic to ferromagnetic resonance occurs in the concentration range of $x=0.01 \div 0.015$. No dramatic changes in the character of the spectrum is seen. Both resonances are characterized by the single resonance line with $g$-factor $g=2$ and the linewidth of about $20 \mathrm{mT}$. In both cases the magnetic anisotropy is very small, it does not exceed $3 \mathrm{mT}$ when comparing the resonance position for perpendicular and in-plane orientation of external magnetic field. The difference between phases is seen in the line amplitude and its temperature dependence. The amplitude of the paramagnetic resonance is smaller and decreases with an increase in temperature according to Curie-Weiss law. The amplitude of the ferromagnetic resonance is stronger, in a low temperature range it is weakly temperature dependent and sharply decreases below the Curie point.

The small magnetic anisotropy, smaller than expected anisotropy caused by the shape anisotropy for a layered sample (originating from the dipole-dipole 
coupling) indicates that the spatial distribution of the ferromagnetic phase is not homogeneous. It does not uniformly fill the whole sample volume but forms an irregular percolated structure.

Generally the ferromagnetic resonance shows that the total magnetic moment originates from the localized $S=5 / 2$ spins of $\mathrm{Mn}^{2+}$ ions. No resonance which could be related to free carriers and no influence of the magnetic moment of holes on the resonance frequency of Mn moment is observed. It means that the localized holes form a paramagnetic system, characterized by a very broad magnetic resonance and that the actual magnetization of holes follows the precession of the Mn moment being anti-parallel to each other.

\section{Ferrimagnetic resonance}

At the Mn concentration of about $x \approx 0.03$ the insulator to metal transition occurs [2, 9]. This Mott-Hubbard transition is accompanied by the change of the magnetic structure which is evidenced by the change of the character of magnetic resonance. As it is shown in Fig. 1 the resonance in metallic phase is very different from that observed in the insulating $\mathrm{Ga}_{1-x} \mathrm{Mn}_{x} \mathrm{As}$. In this chapter we shall argue that the complex anisotropic spectrum corresponds to the ferrimagnetic resonance [22] and proves the occurrence of two macroscopic moments. The isotropic moment of localized $\mathrm{Mn}^{2+}$ spins, characterized by $g=2$, is antiferromagnetically coupled to very anisotropic moment of conducting holes which is characterized by a smaller $g$-factor $[9,11,12]$.

The analysis of the position of the resonances seen in the high field in Fig. 1e designates them as the spin wave resonance. In the presented spectrum the resonance of the uniform mode at $B=0.64 \mathrm{~T}$ corresponds to the high field edge of the spin wave resonances. The evaluation of the magnetic stiffness [6] leads to the mean value of the exchange $\mathrm{Mn}-\mathrm{Mn}$ to be equal $J / k_{\mathrm{B}}=4 \mathrm{~K}$. This is in the well accordance with the Curie temperature observed for this sample $T_{\mathrm{c}}=80 \mathrm{~K}$.

The observation of the spin wave resonances shows that if the exchange interaction is mediated by extended hole states with a small Fermi energy the continuous media models can be successfully applied [5, 6]. The chemical disorder caused by the random distribution of Mn spins is well averaged and the long spin wave can easily propagate for macroscopic distances.

As it is seen from the comparison of spectra (e) and (f) in Fig. 1 the resonance strongly depends on the direction of the applied magnetic field. The position of the resonance of the uniform mode changes from the field $B=0.64 \mathrm{~T}$ for perpendicular to $B=0.3 \mathrm{~T}$ for in-plane orientation. The difference of the resonant fields corresponds to the axial anisotropy field. The sign of the anisotropy field shows that the in-plane is the easy direction of the magnetization. The corresponding $g$-factor measured at $T=2 \mathrm{~K}$ and frequency $9.4 \mathrm{GHz}$ is $g=1.45$.

The $g$-factor as well as the anisotropy field strongly are different for various samples and they depend on the temperature and on the applied magnetic field 
(i.e. on the microwave frequency used in the measurement). With the temperature increase the anisotropy field increases to the maximum at $T \approx 20 \mathrm{~K}$ and decreases at a higher temperature. For the sample discussed the $g$-factor increases with temperature and at $T>40 \mathrm{~K}$ saturates at a value of $g \approx 1.7$. At high magnetic field the $g$-factor for the discussed sample is $g \approx 1.95$. The discussed parameters, in particular, their values at low temperature considerably change from sample to sample.

As well the observed large magnetic anisotropy as the $g$-factor, which is very different from $g=2$, show that the observed magnetic moment cannot originate from spins of the $\mathrm{Mn}^{2+}$ ions only [22]. The observed resonance cannot also be related to the neutral donor state $\left(g=2.77^{19}\right)$ or the spin of $d^{4}$ configuration of $\mathrm{Mn}^{3+}$ ion. On the other hand, all the experimental data presented well confirm the assumption that five $d$ electrons of Mn core form a deep energy state with the $S=5 / 2$ spin which is coupled to the magnetic moment of effective mass holes. The peculiarity of the metallic phase is that the conducting holes form their own macroscopic magnetic moment and the whole system have the properties of the ferrimagnet. When the two subsystems are strongly coupled then the only mode of the ferrimagnetic resonance which can be observed at low magnetic field is, so-called, the Zeeman mode. The frequency ( $g$-factor) and the anisotropy field of such mode are the mean values of the contributing magnetic moments [22]. The coupling of the isotropic moment of $\mathrm{Mn}^{2+}$ spins with the strongly anisotropic moments of conducting holes results in the anisotropic moment with $g<2$ which is experimentally observed. The dependence of the ferrimagnetic resonance on the external parameters reflects the dependence of the ratio of the magnitudes of the contributing moments.

The observation of the ferrimagnetic resonance does not mean only that both spin subsystems are polarized by the $p-d$ exchange. Such polarization is expected in ferromagnetic phase as well. The formation of the macroscopic moment in ferrimagnetic phase means that there is an effective exchange interaction between conducting holes which is strong enough to result in the magnetic order of holes. Here the ordered structure means that all spins precess in a coherent way, with the common precession phase.

\section{Reentrance to the ferromagnetic phase}

For the Mn concentration $x>0.06$ the alloys of $\mathrm{Ga}_{1-x} \mathrm{Mn}_{x}$ As are insulators. This reentrance to the insulating phase is accompanied with re-appearance of the ferromagnetic phase, similar to that which is observed for insulating samples with $x<0.03$. The resonance line is isotropic and occurs at resonance field corresponding to $g=2$. This re-appearance of the ferromagnetic phase in strongly doped, insulating alloys, indicates the direct relation between magnetic and electric properties, i.e. on the role of carriers in magnetic interactions. 


\section{Conclusions}

All the data presented in the paper prove that the $\mathrm{Mn}$ ions occur in $3 d^{5}$ configuration corresponding to $\mathrm{Mn}^{2+}$ ions while the holes occupy the effective mass states: the impurity band for insulating [7, 13] and the valence band states in metallic phase $[5,6]$. The correlation of the electric and the magnetic properties strongly indicates that in $\mathrm{Ga}_{1-x} \mathrm{Mn}_{x}$ As the effective mass holes [5-14], but not the double exchange [15] are responsible for the transfer of the exchange interaction between localized Mn spins. Occurrence of the ferrimagnetic phase shows that the $p-d$ coupling between the $\mathrm{Mn}$ and the extended hole spins leads to the indirect exchange between holes.

\section{Acknowledgments}

We thank French-Polish program Polonium and EC grant No. HPRI-1999-CT-40013. This work has been partially (Z.W.) supported by the EC grant No. HPRI-1999-CT-00030.

\section{References}

[1] M. Tanaka, J. Vac. Sci. Technol. B 16, 2267 (1998).

[2] H. Ohno, J. Magn. Magn. Mater. 200, 110 (1999).

[3] Y. Ohno, D.K. Young, B. Beschoten, F. Matsukura, H. Ohno, D.D. Awschalom, Nature 402, 790 (1999).

[4] H. Ohno, D. Chiba, F. Matsukura, T. Omiya, E. Abe, T. Dietl, Y. Ohno, K. Othani, Nature 408, 944 (2000).

[5] T. Dietl, H. Ohno, F. Matsukura, J. Cibert, D. Ferrand, Science 287, 1019 (2000).

[6] J. König, H.-H. Lin, A.H. MacDonald, Phys. Rev. Lett. 84, 5628 (2001).

[7] V.I. Litvinov, V.K. Dugajev, Phys. Rev. Lett. 86, 5593 (2001).

[8] T. Dietl, H. Ohno, F. Matsukura, Phys. Rev. B 63, 195205 (2001).

[9] T. Dietl, Semicond. Sci. Technol. 17, 377 (2002).

[10] M. Abolfath, T. Jungwirth, J. Brum, A.H. MacDonald, Phys. Rev. B 63, 54418 (2001).

[11] T. Dietl, J. König, A.H. MacDonald, Phys. Rev. B 64, 241201(R) (2001).

[12] J. König, T. Jungwirth, A.H. MacDonald, Phys. Rev. B 64, 184423 (2001).

[13] M. Berciu, R.N. Bhatt, Phys. Rev. Lett. 87, 107203 (2002).

[14] J. Schliemann, A.H. MacDonald, Phys. Rev. Lett. 88, 137201 (2002).

[15] K. Sato, H. Katayama-Yoshida, Semicond. Sci. Technol. 17, 367 (2002).

[16] O.M. Fedorych, E. Hankiewicz, Z. Wilamowski, J. Sadowski, Phys. Rev. B 66, 045201 (2002).

[17] J. Sadowski, J.Z. Domagała, J. Bąk-Misiuk, S. Koleśnik, M. Sawicki, K. Świątek, J. Kanski, L. Ilver, V. Ström, J. Vac. Sci. Technol. B 18, 1697 (2000). 
[18] N. Almeleh, B. Goldstein, Phys. Rev. 128, 1568 (1962).

[19] J. Schneider, U. Kaufmann, W. Wilkening, M. Baeumler, F. Köhl, Phys. Rev. Lett. 59, 240 (1987).

[20] J. Szczytko, W. Mac, A. Twardowski, F. Matsukura, H. Ohno, Phys. Rev. B 59, 12935 (1999).

[21] J. Szczytko, A. Twardowski, K. Świątek, M. Palczewska, M. Tanaka, T. Hayashi, K. Ando, Phys. Rev. B 60, 8304 (1999).

[22] S. Foner, in: Magnetism, Eds. G.T. Rado, H. Suhl, Academic Press, London 1963, p. 381 . 